University of Nebraska - Lincoln

DigitalCommons@University of Nebraska - Lincoln

\title{
Efficacy of Proximity Loggers for Detection of Contacts Between Maternal Pairs of White-Tailed Deer
}

\author{
Ryan Walrath \\ University of Wisconsin, ryandwalrath@gmail.com \\ Timonthy R. VanDeelen \\ University of Wisconsin \\ Kurt C. VerCauteren \\ USDA-APHIS-Wildlife Services, kurt.c.vercauteren@usda.gov
}

Follow this and additional works at: https://digitalcommons.unl.edu/icwdm_usdanwrc

Part of the Life Sciences Commons

Walrath, Ryan; VanDeelen, Timonthy R.; and VerCauteren, Kurt C., "Efficacy of Proximity Loggers for Detection of Contacts Between Maternal Pairs of White-Tailed Deer" (2012). USDA Wildlife Services - Staff Publications. 1607.

https://digitalcommons.unl.edu/icwdm_usdanwrc/1607

This Article is brought to you for free and open access by the U.S. Department of Agriculture: Animal and Plant Health Inspection Service at DigitalCommons@University of Nebraska - Lincoln. It has been accepted for inclusion in USDA Wildlife Services - Staff Publications by an authorized administrator of DigitalCommons@University of Nebraska - Lincoln. 
Original Article

\title{
Efficacy of Proximity Loggers for Detection of Contacts Between Maternal Pairs of White-Tailed Deer
}

\author{
RYAN WALRATH, ${ }^{\mathbf{1 , 2}}$ Department of Forest, Wildlife Ecology, 1630 Linden Drive, 226 Russell Labs, University of Wisconsin, Madison, \\ WI 53706, USA \\ TIMOTHY R. VAN DEELEN, Department of Forest, Wildlife Ecology, 1630 Linden Drive, 226 Russell Labs, University of Wisconsin, Madison, \\ WI 53706, USA \\ KURT C. VerCAUTEREN, United States Department of Agriculture, Animal and Plant Health Inspection Service, Wildlife Services, \\ National Wildlife Research Center, 4101 LaPorte Avenue, Fort Collins, CO 80521, USA
}

\begin{abstract}
Contact frequency and duration estimates between individuals are important to understanding the behavioral ecology of wildlife species and the epidemiology of infectious diseases. A new technology uses proximity data loggers to record time and duration of contacts. We conducted an experiment at Sandhill Wildlife Management Area, located near Babcock, Wisconsin (USA) to compare probabilities of detecting intraspecific contacts among white-tailed deer (Odocoileus virginianus) maternal pairs (dams/fawns) based on detections from proximity loggers deployed on collars versus those obtained from direct observation. We defined 5 discrete probabilities of detection of a contact in terms of $P$ (probability of detection by a single proximity logger) and $V$ (probability of detection by visual observer) and estimated $P$ and $V$ by minimizing the Kullback-Liebler distance between distributions of theoretical probabilities and observed distributions in experimental data. We used parametric jackknifing to estimate means and variances for $P$ and $V$. Mean estimates of $P$ and $V$ were $0.64(95 \% \mathrm{CI}=0.62-0.67)$ and $0.34(0.32-0.35)$, respectively. Estimates of $P$ and $V$ enabled the calculation of the probability that an encounter was undetected by both proximity loggers and the visual observer, which was $0.09(95 \% \mathrm{CI}=0.073-0.094)$. Estimates of $P$ and $V$ provide estimates of nondetection bias for future studies that use proximity loggers to estimate frequencies of encounters and help quantify the usefulness of this technology relative to visual observation. Management concerns such as chronic wasting disease and bovine tuberculosis could be better understood and addressed by using proximity loggers because they are better able to quantify close contact than conventional methods such as radiotelemetry or Global Positioning System telemetry. (c) 2011 The Wildlife Society.
\end{abstract}

KEY WORDS contact rates, data logging, detection bias, efficacy, Odocoileus virginianus, proximity loggers, radiocollars, UHF, VHF, white-tailed deer.

Presence of disease in wildlife complicates management because wildlife may freely move across landscapes and interact with conspecifics and individuals of other species, thereby spreading infection (Weller 2006, Ward et al. 2009). Frequency and duration of contacts between individuals represent important aspects of a species' behavioral ecology (Prange et al. 2006, Schauber et al. 2007). Contact rates also influence epidemiology of infectious diseases (Loveridge and Macdonald 2001, Totton et al. 2002, Prange et al. 2006, Schauber et al. 2007). The effect of contact frequency and duration in many wildlife species (e.g., ungulates) is complex because dynamic social systems result in variation in contact rates as functions of age, kinship, and social position. Examples of recent disease outbreaks in ungulates include

Received: 22 December 2010; Accepted: 7 August 2011;

Published: 28 October 2011

${ }^{1}$ E-mail: ryandwalrath@gmail.com

${ }^{2}$ Present Address: 30 Ochiltree Avenue, Glasgow G13 1LH, UK. chronic wasting disease (CWD; Mathiason et al. 2006; VerCauteren et al. 2007a, b, $c$; Thompson et al. 2008), bovine tuberculosis (TB; Ramsey et al. 2002; Palmer et al. 2004; VerCauteren et al. 2007b, $c$; Thompson et al. 2008), epizootic hemorrhagic disease (Gaydos et al. 2002), and brucellosis (Cross et al. 2007, Thompson et al. 2008). Increased direct and indirect contact rates increase the potential for pathogen transmission in both density- and frequencydependent transmission patterns (McCallum et al. 2001, Begon et al. 2002, Turner et al. 2003, Hone and Donnelly 2008, Smith et al. 2009). Wildlife managers would be able to improve management and decrease the occurrence of disease outbreaks if the effect of contact rates on pathogen transmission was better understood (Corner et al. 2003, Miller et al. 2003, McCallum 2008).

Current methods for estimating frequency and duration of contact between individuals include visual observation (Grenier et al. 1999, Totton et al. 2002), radiotelemetry (White and Harris 1994, Caley et al. 1998, Ramsey et al. 
2002, White et al. 2003, Ji et al. 2005), Global Positioning System (GPS) telemetry (Schauber et al. 2007, Kjaer et al. 2008), and electronic proximity loggers (Ji et al. 2005, Prange et al. 2006). Use of visual observations usually are limited to species that are easily visible (Schaller 1972, Prange et al. 2006) and commonly are obtained at sites where activity is concentrated and observation is convenient, such as natural (Tevis 1947, Reimchen 1998) or artificial feeding sites (Sharp and Sharp 1956, Grenier et al. 1999, Totton et al. 2002), thereby potentially producing biased observations (Côté 2000). Location error in radiotelemetry and GPS telemetry may prevent these technologies from producing the fine-scale spatial and temporal resolution needed in disease studies; hence, contacts must be inferred from close associations in space and time. For example, associations in space and time have been inferred from relatively coarse observations wherein contact is defined as occurring when 2 radiocollared individuals are located within $25-100 \mathrm{~m}$ of each other (White and Harris 1994, Gehrt and Fritzell 1998) or when GPS-instrumented individuals are within 5-25 m (Kjaer et al. 2008). Although GPS telemetry collars are becoming more precise, with mean location errors as low as $10.5 \mathrm{~m}(\mathrm{SE}=0.22)$, confounding factors such as topography, obstruction by overhead vegetation, orientation, and position of collars can cause large $(\bar{x}=17.0 \mathrm{~m}, \mathrm{SE}=1.72)$ positional errors (D'eon and Delparte 2005).

In most applications of GPS telemetry and radiotelemetry, the number of locations observed for animal per unit time (e.g., day, week) is constrained by battery life (e.g., GPS) or the logistics of having technicians in the field generating triangulation data. Estimated locations represent discrete points in time punctuating longer sequences of time when locations cannot be estimated, and, therefore, are unknown. Longer time sequences in GPS studies greatly complicate detecting close contact between individuals because the timing of location estimates for target individuals needs to be carefully synchronized. Traditional radiotelemetry studies rarely capture simultaneous locations for all individuals; the time between locations of individuals is one of the most important factors when radiotelemetry is used to investigate contact between individuals. Traditional radiotelemetry and GPS telemetry have helped answer many ecological questions but a technology that addresses finer temporal and spatial resolution is needed in order to study close contacts between individuals.

Proximity loggers are a relatively new technology for quantifying contacts between animals. Proximity loggers are electronic devices that emit a unique electronic signal while continuously monitoring and recording the time and duration of signals emitted by other loggers. Proximity loggers overcome many logistical difficulties of quantifying contact rates of secretive, nocturnal, or unobservable species and provide information at a finer temporal and spatial resolution than previous methods. Researchers can define a threshold detection distance (e.g., 0.5-100 m) for logging encounters with errors of 1.1-2.8 $\mathrm{m}$ when the detection distance is set around $1.0 \mathrm{~m}$ (Prange et al. 2006), which gives this technology the necessary spatial and temporal resolution needed to quantify rates of close contact between individuals. Previous research using proximity loggers has been limited to laboratory studies of performance, and to one field study of raccoons (Procyon lotor; Prange et al. 2006); the proximity loggers proved reliable in both studies. Efficacy, defined as the ability of proximity loggers to detect actual contacts, however, has not been reported. Detection bias between proximity loggers, a key component when considering error, has not been quantified.

Our goal was to estimate the capability and efficacy of recently developed proximity loggers for recording contacts between instrumented individuals by comparing data collected from proximity loggers and a visual observer. Combining data collection methods provided an avenue for us to investigate detection bias of proximity loggers and visual observers, an important application for ecological studies. We present results on the efficacy of proximity loggers deployed on wild-caught, captive white-tailed deer (Odocoileus virginianus) during an experimental study and a method for quantifying proximity-logger detection bias.

\section{STUDY AREA}

Our study occurred during winter (Dec-Apr) 2007-2008 and 2008-2009 at Sandhill Wildlife Management Area (SWMA), located near Babcock (Wisconsin, USA). Sandhill Wildlife Management Area was a $37 \mathrm{~km}^{2}$ research facility that was maintained by the Wisconsin Department of Natural Resources and was surrounded by a $2.7 \mathrm{~m}$ high, deer-proof fence. The property contained flat, marshy land interspersed with oak (Quercus spp.), aspen (Populus spp.), and jack-pine (Pinus banksiana) forests.

Observations occurred in an experimental deer pen maintained within SWMA (Fig. 1). The pen consisted of a 3.0-m deer-proof fence enclosing a rectangular area of roughly 2.4 ha. The interior was subdivided by deer-proof fencing to create 3 rectangular-shaped interior pens of roughly 0.8 ha each. All interior and exterior fencing was covered by opaque shade cloth.

\section{METHODS}

\section{Proximity Logging System}

Prange et al. (2006) provided a detailed description of contact detection using proximity loggers. In short, proximity loggers use a short-range data link to communicate using transmitters emitting ultra high frequency (UHF) signals coupled with scanning receivers that have a detection distance that is adjustable by the user. Proximity loggers were attached to individual vinyl collars and also contained very high frequency (VHF) transmitters so the collars could be located using conventional radiotelemetry techniques. The proximity loggers (hardware version 1.8; Sirtrack Ltd., Havelock North, New Zealand) were designed to log the identity, time, date, and duration of other instrumented individuals coming within the detection range. Hardware version 1.8 currently is marketed to wildlife researchers but with updated internal programming. 


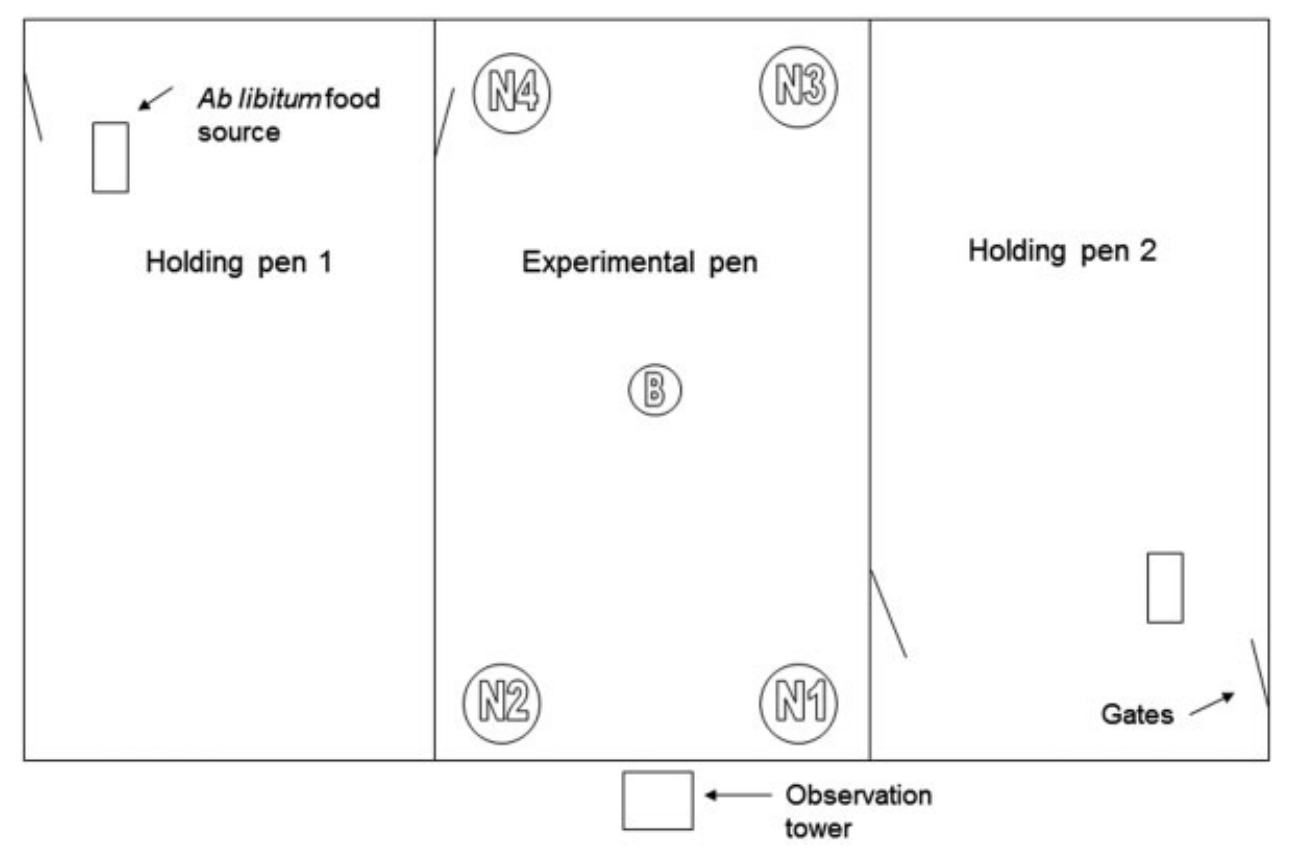

Figure 1. Diagram of research pen containing 30.8 -ha sections for studying contacts between individual white-tailed deer at Sandhill Wildlife Management Area (Babcock, WI, USA) 2007-2008. Holding pens 1 and 2 were used to house maternal pairs while a second pair was being trapped, and to acclimate deer to the pen environment. Contacts were measured in the central experimental pen.

Hardware version 1.8 functioned as follows: 1) each record consisted of 5 data fields: entry number (i.e., record number), ID number of the contacted logger, date (dd/mm/yyyy), time contact was initiated (hr:min:s), and contact duration in seconds; 2) user-defined parameters included current date (dd/mm/yyyy) and time (hr:min:s), the identification number to be broadcasted (1-250), separation time required for defining discrete contacts (1-255 s), UHF transmitter output power (which determined the detection distance: 0 [strongest]-62 [weakest]), VHF beacon pulse rate (5$200 \mathrm{pulses} / \mathrm{min}$ ), and user information (settings that facilitated data organization for subsequent analysis [e.g., location, animal ID, etc.]); and 3) data may be downloaded to the interface unit (e.g., laptop, handheld portable computer, etc.).

We defined an encounter as any time a logger communicated with another logger. We defined a separation time of $120 \mathrm{~s}$, thereby making the assumption that any encounters separated by $\geq 120 \mathrm{~s}$ were separate encounters. We assigned each proximity logger in our study a unique identification number. We adjusted the UHF transmitter coefficient of each logger experimentally to achieve a detection distance of $1 \mathrm{~m}$ and to insure that detection distances were as uniform as possible among all loggers (R. Walrath, unpublished data). Individually testing each logger's transmitter coefficient to reflect the desired detection distance reduced potential error from differences in transmitters among loggers (Prange et al. 2006). To determine whether the woven-wire fence of the pen impacted loggers (i.e., by functioning as an antenna) we evaluated whether false positives could occur due to signal interference by incrementally moving a proximity logger away (e.g., from right next to the fence out to $10 \mathrm{~m}$ ) from a stationary proximity logger, both parallel and perpendicular to the fence.
Previous researchers (Prange et al. 2006, Goodman 2007) removed 1-s contacts from analysis to filter out occurrences of broken contacts. We did not remove 1-s contacts because we observed short-duration contacts (e.g., passing, chasing, etc.) that could be informative for research. To get true contact durations between individuals, we used the cumulative duration of overlapping contacts recorded and the assumption that individuals were in contact when $\geq 1$ of the loggers was recording a contact (Appendix). Corrupted records that were easily identifiable due to dates being recorded incorrectly (e.g., 9/9/9999) or encounter durations that were out of proportion to other contact durations (e.g., longer than possible for study time frame) were removed from the data set.

Deer capture and handling.-From January to April, we captured deer on SWMA using rocket-propelled nets (Kilpatrick et al. 1997, Cromwell and Warren 1999, Haulton et al. 2001) or drop-nets (White and Bartmann 1994, Peterson et al. 2003, Jedrzejewski and Kamler 2004) at sites baited with corn, alfalfa pellets, molasses, and apples. We targeted deer whose behavior and relative size suggested they were dam/fawn (maternal) pairs. Distances between trapping locations were maximized in order to decrease potential relatedness between pairs and to enable us to assume pairs would be unfamiliar with each other. Use of maternal pairs was required for a companion study (of the effects of relatedness and food distribution on contact rates [R. Walrath, unpublished data]) but also ensured we would have a range of potential contact frequencies for this study of logger efficacy.

We anesthetized captured deer with an intramuscular injection of xylazine $\mathrm{HCl}(2.5 \mathrm{mg} / \mathrm{kg}$; IVX Animal Health, Inc., St. Joseph, MO) and Telozol ${ }^{\circledR}$ (1:1 tiletamine hydrochloride and zolazepam hydrochloride association; 
3.5-5.0 mg/kg; Fort Dodge Animal Health, Fort Dodge, IA) for transport to the research pen and attachment of proximity loggers. Once deer were placed in the research pen and $80 \mathrm{~min}$ had passed, we reversed the xylazine with Tolazoline $\mathrm{HCl}$ (3 mg/kg; Akorn, Inc., Decatur, IL; Amass and Drew 2007). While deer were anesthetized, we blindfolded them, painted unique identifying symbols on their sides with nontoxic sheep-marking paint (Etro-Mark ${ }^{\mathrm{TM}}$, Clayton, NM) and monitored their vital signs (e.g., heart rate, respiration, and body temp). We visually monitored deer for a minimum of $1 \mathrm{hr}$ after they could stand on their own.

Following the trials, we retrieved data loggers by darting deer with 2-cc barbed darts (Pneu-Dart, Inc., Williamsburg, PA) containing the same mixture used after initial capture. After we removed the proximity loggers, we reversed the xylazine with the same mixture as before and released deer back onto SWMA. Deer capture and handling methods were approved by the University of Wisconsin-Madison Institutional Animal Care and Use Committee (protocol no. A1272).

\section{Data Collection (Visual Observations)}

After capture, we held each maternal pair separately in 1 of 2 holding pen sections of the research pen (Fig. 1). A trial (i.e., 3 days or $72-\mathrm{hr}$ period of data collection) began when 4 unique animals (i.e., 2 maternal pairs) were caught. All animals were fitted with proximity loggers and we placed both pairs into the experimental pen section (Fig. 1) for data collection. We then conducted 62 -hr observation periods on the 4 individuals, $1 \mathrm{hr}$ before and after sunrise and sunset (i.e., $2 \mathrm{hr}, 2$ times daily) for 3 days, producing 12 total hr of observation for each set of animals. The study consisted of 6 3 -day trials that produced $72 \mathrm{hr}$ of visual observation data and $432 \mathrm{hr}$ of proximity logger data for all groups.

We conducted observations from an enclosed blind on an elevated platform (Big Game Treestands, Windom, MN) located $1 \mathrm{~m}$ outside the pen perimeter and overlooking the experimental pen. We painted each proximity logger a different color, which (in combination with the painted symbol on the side of the deer) aided the visual observer in correctly identifying individuals during encounters. Occasionally deer would exhibit abnormal behaviors (e.g., gathering in corners farthest from observation tower) in reaction to the observer approaching the pen and climbing into the observation blind. The observer entered the blind 15 min before recording was initiated to allow deer to resume previous behaviors. Data recorded by the observer included date, logger IDs of deer involved in encounter, encounter start time, duration to the nearest $15 \mathrm{~s}$ and behavior associated with encounter. We defined encounters as occurring when deer were within $1 \mathrm{~m}$ of each other, because this distance approximated the body length of a deer and enabled the visual observer to use body length as a cue for identifying an encounter. Furthermore, bacterial and viral pathogens are realistically transmitted at distances $\leq 1 \mathrm{~m}$ (Sauter and Morris 1995, Gaydos et al. 2002).

\section{Analysis of Contacts Recorded by Proximity Loggers}

We tested for differences in counts of encounters relative to relatedness (related, not related) using paired $t$-tests (PROC TTEST, SAS 9.2; SAS Institute, Inc., Cary, NC). Observations were encounters detected by the observer or by the proximity loggers. Observations were paired by trial $(N=6)$. We tested for differences in durations of detected contacts relative to relatedness using generalized linear models (PROC GLM). Effects were relatedness, trial, and their interaction.

Initial data analysis revealed several encounters detected by one (but not both) proximity loggers, indicating that individual proximity loggers did not detect encounters with perfect certainty, thus underestimating actual frequency of encounters. We used direct observations to estimate the probability that a logger detected an encounter.

There were 5 possible ways to detect an encounter when using data from proximity loggers and our visual observations (Table 1$)$. These 5 cases $\left(C_{i}, i=1 \ldots 5\right)$ can be represented as expected probabilities $\left(\hat{C_{i}}\right)$ defined in terms of $P$ and $V$, where $P=$ the probability that a logger detects an encounter and $V=$ the probability that the visual observer detects an encounter. Counts of $C_{i}$ represent a distribution of observations obtained for each of the 5 ways for each observation period, whereas $\hat{C}_{i}$ represents a distribution of discrete probabilities corresponding to each $C_{i}$. Estimation of $P$ and $V$, thus, required choosing values for $P$ and $V$ that jointly minimize the statistical distance between the distributions of $C_{i, j}$ and $\hat{C_{i}}$, where $i$ indexes case and $j$ indexes observation period.

The Kulback-Liebler distance (KL) is a commonly used measurement of the statistical distance between distributions (Iverson et al. 2004); thus, we used PROC NLP (SAS 9.2; SAS Institute, Inc.) to minimize

$$
\mathrm{KL}=\sum_{i=1}^{5}\left[\left(C_{i}-\hat{C}_{i}\right) \ln \left(\frac{C_{i}}{\hat{C}_{i}}\right)\right]
$$

Table 1. Cases observed during visual observation (VO) of interacting proximity loggers deployed on white-tailed deer in a 0.8-ha pen at Sandhill Wildlife Management Area (Babcock, Wisconsin, USA), 2007-2008.

\begin{tabular}{lc}
\hline Observed case $\left(C_{\boldsymbol{i}}\right)$ & Estimated probability of $\boldsymbol{C}_{\boldsymbol{i}}$ in terms of $\boldsymbol{P}$ and $\boldsymbol{V}^{\text {a }}\left(\hat{\boldsymbol{C}_{\boldsymbol{i}}}\right)$ \\
\hline$C_{1}=$ cases detected by both loggers and the VO & $\hat{C_{1}}=P^{2} V$ \\
$C_{2}=$ cases detected both loggers but not by VO & $\hat{C}_{2}=P^{2}(1-V)$ \\
$C_{3}=$ cases detected by one logger and the VO & $\hat{C_{3}}=P(1-P) V$ \\
$C_{4}=$ cases detected by one logger but not by VO & $\hat{C}_{4}=P(1-P)(1-V)$ \\
$C_{5}=$ cases not detected by a logger but by VO & $\hat{C_{5}}=(1-P)^{2} V$ \\
\hline
\end{tabular}

${ }^{\text {a }} P=$ probability that a logger detects an encounter, $V=$ probability that the visual observer detects the encounter. 
subject to

$$
0<P<1.0
$$

and

$$
0<V<1.0
$$

where, $C_{i}$ is the counts of case $i$ (Table 1 ) and $\hat{C_{i}}$ the expected probabilities of $C_{i}$ in terms of $P$ and $V$ (Table 1 ).

We used parametric jackknifing (Efron 1981) to estimate means and variances for $P$ and $V$. Parametric jackknifing is a resampling technique wherein $N$ pseudo-samples are created from the original data set by sequentially removing one observation. We estimated $P$ and $V$ for each of the $N$ pseudo-samples, and mean and variances were calculated from $P_{i}$ and $V_{i}(i=1 \ldots N)$.

\section{RESULTS}

We captured 21 and 11 deer during the 2007 and 2008 field seasons, respectively. We captured 17 deer with drop-nets and 15 with rocket-nets. Eight deer were incidental captures; 24 deer (12 maternal pairs) were subjects for the experiment (i.e., 6 trials with 4 unique deer/trial). We held deer for a mean of 17 days ( $\mathrm{SD}=9$ days) from the time the first pair was captured until both pairs were released back onto SWMA. We repeatedly used 10 proximity loggers that were randomly allocated to an animal during the study (i.e., proximity loggers were deployed more than once); 1 logger failed to download data in the field and was sent to the manufacturer where data were downloaded successfully, but the logger was compromised.

One observer (R.W.) conducted $12 \mathrm{hr}$ of direct observations for each of 6 trials evaluating proximity loggers among maternal pairs. We found the fence did not affect functionality (i.e., deflecting signals or acting as an antenna) of the proximity loggers.

Our observer recorded 500 encounters $(\bar{x}=142.9 /$ trial, $\mathrm{SE}=27.3)$, whereas the proximity loggers detected 774 encounters $(\bar{x}=221.1, \mathrm{SE}=28.5)$. Counts of inter- and intramaternal pair encounters detected did not differ for encounters detected by the observer $\left(t_{5}=1.33, P=0.24\right)$ or by the proximity loggers $\left(t_{5}=-0.21, P=0.84\right)$. Total duration of observed encounters was $76,445 \mathrm{~s}(\bar{x}=152.0$, $\mathrm{SE}=224.3)$, whereas total duration of encounters recorded by proximity loggers was $112,908 \mathrm{~s}(\bar{x}=221.1, \mathrm{SE}=28.5)$. Durations of encounters recorded by our observer differed with respect to relatedness (Type 3 sums of squares, $\left.F_{1}=43.3, P<0.001\right)$, trial $\left(F_{5}=3.59, P=0.003\right)$, and the interaction between relatedness and trial $\left(F_{5}=5.63\right.$, $P<0.001)$. Durations of contacts for related individuals (least-squares $\bar{x}=221.8$ ) were longer than for unrelated individuals (least-squares $\bar{x}=88.1$, Tukey-Kramer test, $P<0.001)$. Similarly, durations of encounters recorded by proximity loggers differed with respect to relatedness $\left(F_{1}=60.54, P<0.001\right)$, trial $\left(F_{5}=4.28, \quad P<0.001\right)$, and the interaction between relatedness and trial $\left(F_{5}=4.33, P<0.001\right)$. Durations of contacts for related individuals (least-squares $\bar{x}=370.8$ ) were longer than for unrelated individuals (least-squares $\bar{x}=94.2$, TukeyKramer test, $P<0.001)$.

Counts of encounter cases $\left(C_{i}\right)$ indicated that most encounters were detected by both loggers but not by the visual observer (Table 2). Estimation of $P_{i}$ and $V_{i}$ were remarkably consistent across the 6 psuedo-samples created for jackknifing (Table 3). Consequently, the confidence intervals were relatively narrow. Jackknifed estimates of mean $P$ and $V$ were 0.64 (95\% CI $=0.62-0.67)$ and $0.34(0.32-0.35)$, respectively. Estimates of $P$ and $V$ enabled calculation of the probability that an encounter was undetected by both proximity loggers and the visual observer $\left[(1-P)^{2}(1-V)\right]$. Variance of the quantity $(1-P)^{2}(1-V)$ was estimated using Monte Carlo simulation from 500 random draws of $P_{i} \sim N(0.645,0.0138)$ and $V_{i} \sim N(0.337,0.010)$. Hence, the estimated probability of an encounter being missed by both proximity loggers and the visual observer was 0.09 (95\% CI $=0.073-0.094)$.

\section{DISCUSSION}

Given the detection distance we defined ( $\leq 1 \mathrm{~m})$, the efficacy of proximity loggers deployed to detect close contacts between white-tailed deer was 64\% $(P)$ and was significantly better than that of a visual observer in a controlled setting $(34 \%, V)$. Even so, roughly $9 \%$ of contacts were undetected by either proximity logger or by observer, and our data

Table 2. Distribution of cases where an encounter between any combination of 2 white-tailed deer was detected by the 2 deer's proximity loggers and a visual

\begin{tabular}{|c|c|c|c|c|c|c|}
\hline \multirow[b]{2}{*}{ Trial } & \multicolumn{6}{|c|}{ Case (\%) } \\
\hline & $\overline{\mathrm{C}, \mathrm{C}, \mathrm{VO}}$ & $\mathrm{C}, \mathrm{C}$ & C,VO & $\mathrm{C}$ & VO & Total count \\
\hline 1 & 0.05 & 0.45 & 0.02 & 0.20 & 0.27 & 683 \\
\hline 2 & 0.06 & 0.38 & 0.03 & 0.23 & 0.30 & 517 \\
\hline 3 & 0.06 & 0.52 & 0.02 & 0.21 & 0.19 & 389 \\
\hline 4 & 0.10 & 0.39 & 0.04 & 0.23 & 0.23 & 444 \\
\hline 5 & 0.06 & 0.44 & 0.03 & 0.22 & 0.26 & 400 \\
\hline 6 & 0.14 & 0.45 & 0.04 & 0.26 & 0.11 & 536 \\
\hline Mean & 0.08 & 0.44 & 0.03 & 0.23 & 0.22 & \\
\hline Lower 95\% CI & 0.01 & 0.34 & 0.01 & 0.18 & 0.09 & \\
\hline Upper 95\% CI & 0.14 & 0.54 & 0.05 & 0.27 & 0.36 & \\
\hline
\end{tabular}
observer during an experiment at Sandhill Wildlife Management Area (Babcock, Wisconsin, USA), 2007-2008.

${ }^{a}$ Key: $\mathrm{C}$, encounter detected by one proximity logger; VO, detection by visual observer; C,C,VO, detection by both proximity loggers and the visual observer. 
Table 3. Estimates of mean probability that an encounter between whitetailed deer during an experiment at Sandhill Wildlife Management Area (Babcock, Wisconsin, USA), 2007-2008, was detected by a proximity logger $(P)$ and the mean probability that an encounter was detected by a visual observer $(V)$. Row entries indicate estimates of $P_{i}$ and $V_{i}$ calculated for pseudo-samples created for jackknifing (Efron 1981). Psuedo-samples were created by sequentially excluding an observation from the data set.

\begin{tabular}{lcc}
\hline Observation excluded & $\boldsymbol{P}_{\boldsymbol{i}}$ & $\boldsymbol{V}_{\boldsymbol{i}}$ \\
\hline 1 & 0.65 & 0.33 \\
2 & 0.66 & 0.33 \\
3 & 0.64 & 0.35 \\
4 & 0.65 & 0.33 \\
5 & 0.65 & 0.33 \\
6 & 0.62 & 0.35 \\
Mean & 0.64 & 0.34 \\
Lower 95\% CI & 0.62 & 0.32 \\
Upper 95\% CI & 0.67 & 0.35 \\
\hline
\end{tabular}

suggest that roughly $13 \%\left[(1-P)^{2}\right]$ would be undetected in a field study where visual observation was not possible. We hypothesize that efficacy would increase as detection distance is increased. When detection distance is increased, the volume of the 3-dimensional space surrounding the proximity logger where detection is reliable accelerates relative to the increase in the boundary of that space where detection is marginal. Additional experiments would be needed to evaluate this hypothesis.

Variation in logger transmission and reception strength combined with interactions occurring near the limits of detection will cause differences in the number and duration of contacts detected (Prange et al. 2006). Signal strength could be weaker outside the set range of detection, which could account for some of the variation between proximity loggers' recorded number and duration of contacts, but more research is needed to evaluate this hypothesis. Further, variation in logger transmission and reception could also be influenced by the orientation of the proximity logger (Prange et al. 2006) and attenuation. Signal strength will attenuate more quickly if it has to pass through a medium (e.g., another deer, the neck of the deer with the proximity logger, vegetation, etc.). Attenuation could have the opposite effect, where rather than a medium (e.g., soil) absorbing the radio wavelengths, the medium (e.g., water and snow) could reflect or scatter the wavelengths and potentially increase the chances that proximity loggers outside the detection distance would $\log$ an encounter (i.e., false positive). To reduce the bias caused by attenuation and proximity logger orientation, we recommend estimating correction factors for the expected habitats and environmental conditions in which the proximity loggers will be used.

Reliable methods to estimate contact rates accurately and precisely in a population are critically needed (Schauber et al. 2007). Proximity loggers can detect contacts reliably at distances as close as 1.1-2.8 $\mathrm{m}$ (Prange et al. 2006). Conventional VHF radiotelemetry cannot achieve this spatial resolution. Given the positional error of locations, studies that used VHF collars commonly defined a contact to have occurred when 2 individuals were $\geq 25-100 \mathrm{~m}$ apart (White and Harris 1994, Gehrt and Fritzell 1998,
White et al. 2000, Ramsey et al. 2002, Atwood and Weeks 2003). Global Positioning System locations can be accurate to $\geq 5 \mathrm{~m}$, but positional errors have been reported to be as large as $17.0 \mathrm{~m}$ ( $\mathrm{SE}=1.72$; D'eon and Delparte 2005); however, GPS-collar position, habitat use, and topography may affect both fix success rates and location error (Frair et al. 2004, D'eon and Delparte 2005, D'eon and Serrouya 2005).

Near-continuous operation is needed to measure the number and duration of contacts. Global Positioning System collars and traditional VHF collars do not have the temporal resolution that proximity loggers are able to provide, given their ability to send out a signal every $1.5 \mathrm{~s}$ while simultaneously searching for signals from other loggers. Global Positioning System collars are able to provide information on juxtaposition and location of individuals if they are programmed to record locations at a high enough sampling frequency, but increased sampling frequency results in reduced battery life (Mills et al. 2006). Proximity loggers operate nearly continuously and are better suited to quantify contacts because, unlike GPS collars, there is no programmed interval when data cannot be gathered. Our research demonstrated the importance of near-continuous operation by showing that the significant differences between inter- and intradyad contacts between deer in artificial conditions were not the number of contacts detected but rather the duration of those contacts. The battery life of the proximity loggers used in this study has not been fully analyzed but the battery supplied from the manufacturer was not replaced throughout the 8 months of field work. The battery life may vary between studies due to differences in detection distances, frequency of contacts, climate, and use of VHF transmitters.

Visual observation combined with loggers on maternal pairs enabled us to calculate the probability that an encounter was detected by a proximity logger and the probability that an encounter was detected by a visual observer (Table 3). Each proximity logger's UHF range coefficient was set to detect signals at $1 \mathrm{~m}$, which allowed us to assume detection probability of each logger was equivalent. Even though the probability of a logger detecting another logger $(P)$ was lower than we had hoped, the confidence interval of $P$ was narrow. Despite the fact the proximity loggers performed below our personal expectation, we conclude that the technology (i.e., hardware) of each proximity logger is consistent and precise. We recommend testing loggers in laboratory settings before deployment in the field.

The probability that our visual observer detected an encounter $(V)$ was lower than $P$ but this estimate also was precise despite evidence for variation in contact rates relative to relatedness and trial. The lower visual detection rate could be explained by the visual observer being more conservative in recording a contact than the loggers. The narrow confidence interval (0.09-0.36) may be a result of using the same observer in all trials, thereby eliminating variation due to differences in observers. The reason the observer's detection probability was lower than the logger detection probability may be that there was only one 
observer for 4 deer, which means the observer may have missed encounters taking place in a separate location at the same time. Furthermore, the observer may have missed encounters while recording previous encounters. These events provide evidence that data loggers can be a useful method for collecting data when visual observations are not practical and may generally be more accurate than visual observation.

The differences in detection bias between proximity loggers and visual observation indicate standardizing and comparing detection biases is difficult, particularly when various methods are employed and if the devices are sensitive to calibration. The bi-directional data recording of the proximity loggers provides a method for detection bias correction. Furthermore, the continuous recording of contacts by data loggers provides a more complete data set of encounters, whereas visual observation studies are limited to sites where visual observations are possible (e.g., bait sites and natural mineral licks) and subject to the logistical constraints of having observers make observations in the field. With regard to disease transmissibility, a wild population would, in an ideal situation, need to be nearly saturated with proximity loggers in order to verify all potential vectors were collared, which could be spatially, temporally, and financially difficult unless it was part of a transplant study or isolated (e.g., island) population. Given the aforementioned, proximity loggers provide better data in regard to contact parameters than previous technology. Furthermore, telemetry studies also suffer from populations not being saturated with data recording devices, because few studies are designed to radiocollar an entire population. Given that a population is not likely to be saturated with data collection devices, studies using proximity loggers would be more financially feasible because they are less expensive than other data collection technologies (e.g., GPS collars).

\section{MANAGEMENT IMPLICATIONS}

Proximity loggers are a spatially and temporally precise tool for researchers and managers who need to study contact rates between individual animals. Proximity loggers do a better job of quantifying the number and duration of close contacts than visual observations and our research provided an estimate for the number of close contacts missed. Proximity loggers are useful for research questions where close contact between individuals are important. Diseases such as CWD and TB with direct management implications could be better understood by using proximity loggers in core areas where close contact could play a role in disease transmission. Proximity loggers placed in distinct areas of interest on the landscape (e.g., mineral lick, bait station, watering hole) and on a variety of species of interest could be another scenario where proximity loggers would provide useful data to managers. New technology has previously led the way to new discoveries and provided a mode to better understand natural processes; proximity loggers have the potential to do the same.

\section{ACKNOWLEDGMENTS}

Primary funding was provided by the U.S. Department of Agriculture-Animal and Plant Health Inspection ServicesWildlife Services-National Wildlife Research Center, Fort Collins, Colorado, USA. We thank the Wisconsin Department of Natural Resources and Wisconsin Department of Natural Resources Bureau of Integrated Science Services for additional support and access to our research site. Furthermore, we thank the staff at Sandhill Wildlife Management Area for providing assistance during our trials and the University of Wisconsin-Madison administrative staff and faculty for support and guidance. Finally, we deeply thank M. Watt, volunteers and staff who assisted on this project. Publication of this article does not constitute endorsement or censure of any of the products mentioned by the authors or their employers.

\section{LITERATURE CITED}

Amass, K. D., and M. Drew. 2007. Chemical immobilization of animals: technical field notes. Safe-Capture International, Mt. Horeb, Wisconsin, USA.

Atwood, T. C., and H. P. Weeks. 2003. Spatial home-range overlap and temporal interaction in eastern coyotes: the influence of pair types and fragmentation. Canadian Journal of Zoology-Revue Canadienne De Zoologie 81:1589-1597.

Begon, M., M. Bennett, R. G. Bowers, N. P. French, S. M. Hazel, and J. Turner. 2002. A clarification of transmission terms in host-microparasite models: numbers, densities and areas. Epidemiology and Infection 129: 147-153.

Caley, P., N. J. Spencer, R. A. Cole, and M. G. Efford. 1998. The effect of manipulating population density on the probability of den-sharing among common brushtail possums, and the implications for transmission of bovine tuberculosis. Wildlife Research 25:383-392.

Corner, L. A. L., D. U. Pfeiffer, and R. S. Morris. 2003. Social-network analysis of Mycobacterium bovis transmission among captive brushtail possums (Trichosurus vulpecula). Preventive Veterinary Medicine 59:147-167.

Côté, S. D. 2000. Determining social rank in ungulates: a comparison of aggressive interactions recorded at a bait site and under natural conditions. Ethology 106:945-955.

Cromwell, J. A., and R. J. Warren. 1999. Live-capture and small-scale relocation of urban deer on Hilton Head Island, South Carolina. Wildlife Society Bulletin 27:1025-1031.

Cross, P. C., W. H. Edwards, B. M. Scurlock, E. J. Maichak, and J. D. Rogerson. 2007. Effects of management and climate on elk brucellosis in the greater Yellowstone ecosystem. Ecological Applications 17:957-964.

D'eon, R. G., and D. Delparte. 2005. Effects of radio-collar position and orientation on GPS radio-collar performance, and the implications of PDOP in data screening. Journal of Applied Ecology 42:383-388.

D'eon, R. G., and R. Serrouya. 2005. Mule deer seasonal movements and multiscale resource selection using global positioning system radiotelemetry. Journal of Mammalogy 86:736-744.

Efron, B. 1981. Nonparametric estimates of standard error-the jackknife, the bootstrap and other methods. Biometrika 68:589-599.

Frair, J. L., S. E. Nielsen, E. H. Merrill, S. R. Lele, M. S. Boyce, R. H. M. Munro, G. B. Stenhouse, and H. L. Beyer. 2004. Removing GPS collar bias in habitat selection studies. Journal of Applied Ecology 41:201-212.

Gaydos, J. K., A. B. Allison, B. A. Hanson, and A. S. Yellin. 2002. Oral and fecal shedding of epizootic hemorrhagic disease virus, serotype 1 from experimentally infected white-tailed deer. Journal of Wildlife Diseases 38:166-168.

Gehrt, S. D., and E. K. Fritzell. 1998. Resource distribution, female home range dispersion and male spatial interactions: group structure in a solitary carnivore. Animal Behaviour 55:1211-1227.

Goodman, E. L. 2007. Quantifying interactions in a high-density badger (Meles meles) population. Dissertation, University of York, York, UK. 
Grenier, D., C. Barrette, and M. Crête. 1999. Food access by white-tailed deer (Odocoileus virginianus) at winter feeding sites in eastern Quebec. Applied Animal Behaviour Science 63:323-337.

Haulton, S. M., W. F. Porter, and B. A. Rudolph. 2001. Evaluating 4 methods to capture white-tailed deer. Wildlife Society Bulletin 29:255264.

Hone, J., and C. A. Donnelly. 2008. Evaluating evidence of association of bovine tuberculosis in cattle and badgers. Journal of Applied Ecology 45:1660-1666.

Iverson, S. J., C. Field, W. D. Bowen, and W. Blanchard. 2004. Quantitative fatty acid signature analysis: a new method of estimating predator diets. Ecological Monographs 74:211-235.

Jedrzejewski, W., and J. F. Kamler. 2004. Modified drop-net for capturing ungulates. Wildlife Society Bulletin 32:1305-1308.

Ji, W. H., P. C. L. White, and M. N. Clout. 2005. Contact rates between possums revealed by proximity data loggers. Journal of Applied Ecology 42:595-604.

Kilpatrick, H. J., S. M. Spohr, and A. J. DeNicola. 1997. Darting urban deer: techniques and technology. Wildlife Society Bulletin 25:542-546.

Kjaer, L. J., E. M. Schauber, and C. K. Nielsen. 2008. Spatial and temporal analysis of contact rates in female white-tailed deer. Journal of Wildlife Management 72:1819-1825.

Loveridge, A. J., and D. W. Macdonald. 2001. Seasonality in spatial organization and dispersal of sympatric jackals (Canis mesomelas and C. adustus): implications for rabies management. Journal of Zoology 253:101-111.

Mathiason, C. K., J. G. Powers, S. J. Dahmes, D. A. Osborn, K. V. Miller, R. J. Warren, G. L. Mason, S. A. Hays, J. Hayes-Klug, D. M. Seelig, M. A. Wild, L. L. Wolfe, T. R. Spraker, M. W. Miller, C. J. Sigurdson, G. C. Telling, and E. A. Hoover. 2006. Infectious prions in the saliva and blood of deer with chronic wasting disease. Science 314:133-136.

McCallum, H. 2008. Tasmanian devil facial tumour disease: lessons for conservation biology. Trends in Ecology and Evolution 23:631-637.

McCallum, H., N. Barlow, and J. Hone. 2001. How should pathogen transmission be modeled? Trends in Ecology and Evolution 16:295300.

Miller, R., J. B. Kaneene, S. D. Fitzgerald, and S. M. Schmitt. 2003. Evaluation of the influence of supplemental feeding of white-tailed deer (Odocoileus virginianus) on the prevalence of bovine tuberculosis in the Michigan wild deer population. Journal of Wildlife Diseases 39:84-95.

Mills, K. J., B. R. Patterson, and D. L. Murray. 2006. Effects of variable sampling frequencies on GPS transmitter efficiency and estimated wolf home range size and movement distance. Wildlife Society Bulletin 34:1463-1469.

Palmer, M. V., W. R. Waters, and D. L. Whipple. 2004. Investigation of the transmission of Mycobacterium bovis from deer to cattle through indirect contact. American Journal of Veterinary Research 65:1483-1489.

Peterson, M. N., R. R. Lopez, P. A. Frank, M. J. Peterson, and N. J. Silvy. 2003. Evaluating capture methods for urban white-tailed deer. Wildlife Society Bulletin 31:1176-1187.

Prange, S., T. Jordan, C. Hunter, and S. D. Gehrt. 2006. New radiocollars for the detection of proximity among individuals. Wildlife Society Bulletin 34:1333-1344.

Ramsey, D., N. Spencer, P. Caley, M. Efford, K. Hansen, M. Lam, and D. Cooper. 2002. The effects of reducing population density on contact rates between brushtail possums: implications for transmission of bovine tuberculosis. Journal of Applied Ecology 39:806-818.

Reimchen, T. E. 1998. Nocturnal foraging behaviour of black bears, Ursus americanus, on Moresby Island, British Columbia. Canadian FieldNaturalist 112:446-450.

Sauter, C. M., and R. S. Morris. 1995. Behavioral studies on the potential for direct transmission of tuberculosis from feral ferrets (Mustela furo) and possums (Trichosurus vulpecula) to farmed livestock. New Zealand Veterinary Journal 43:294-300.

Schaller, G. B. 1972. The Serengeti lion: a study of predator-prey relations. University of Chicago, Illinois, USA.

Schauber, E. M., D. J. Storm, and C. K. Nielsen. 2007. Effects of joint space use and group membership on contact rates among white-tailed deer. Journal of Wildlife Management 71:155-163.

Sharp, W. M., and L. H. Sharp. 1956. Nocturnal movements and behavior of wild raccoons at a winter feeding station. Journal of Mammalogy 37:170-177.
Smith, M. J., S. Telfer, E. R. Kallio, S. Burthe, A. R. Cook, X. Lambin, and M. Begon. 2009. Host-pathogen time series data in wildlife support a transmission function between density and frequency dependence. Proceedings of the National Academy of Sciences of the United States of America 106:7905-7909.

Tevis, L., Jr. 1947. Summer activities of California raccoons. Journal of Mammalogy 28:323-332.

Thompson, A. K., M. D. Samuel, and T. R. Van Deelen. 2008. Alternative feeding strategies and potential disease transmission in Wisconsin whitetailed deer. Journal of Wildlife Management 72:416-421.

Totton, S. C., R. R. Tinline, R. C. Rosatte, and L. L. Bigler. 2002. Contact rates of raccoons (Procyon lotor) at a communal feeding site in rural eastern Ontario. Journal of Wildlife Diseases 38:313-319.

Turner, J., M. Begon, and R. G. Bowers. 2003. Modeling pathogen transmission: the interrelationship between local and global approaches. Proceedings of the Royal Society of London Series B: Biological Sciences 270:105-112.

VerCauteren, K. C., P. W. Burke, G. E. Phillips, J. W. Fischer, N. W. Seward, B. A. Wunder, and M. J. Lavelle. 2007a. Elk use of wallows and potential chronic wasting disease transmission. Journal of Wildlife Diseases 43:784-788.

VerCauteren, K. C., M. J. Lavelle, N. W. Seward, J. W. Fischer, and G. E. Phillips. 2007b. Fence-line contact between wild and farmed cervids in Colorado: potential for disease transmission. Journal of Wildlife Management 71:1594-1602.

VerCauteren, K. C., M. J. Lavelle, N. W. Seward, J. W. Fischer, and G. E. Phillips. 2007c. Fence-line contact between wild and farmed white-tailed deer in Michigan: potential for disease transmission. Journal of Wildlife Management 71:1603-1606.

Ward, A. I., K. C. VerCauteren, W. D. Walter, E. Gilot-Fromont, S. Rossi, G. Edwards-Jones, M. Lambert, M. R. Hutchings, and R. J. Delahay. 2009. Options for the control of disease 3: targeting the environment. Pages 147-168 in R. J. Delahay, G. C. Smith, and M. R. Hutchings, editors. Management of disease in wild animals. Springer, Tokyo, Japan.

Weller, R. E. 2006. Risk of disease spread through bioterrorism. Veterinaria Italiana 42:351-367.

White, G. C., and R. M. Bartmann. 1994. Drop nets versus helicopter net guns for capturing mule deer fawns. Wildlife Society Bulletin 22:248-252.

White, P. C. L., and S. Harris. 1994. Encounters between red foxes (Vulpes vulpes)-implications for territory maintenance, social cohesion and dispersal. Journal of Animal Ecology 63:315-327.

White, P. C. L., G. A. Newton-Cross, M. Gray, R. Ashford, C. White, and G. Saunders. 2003. Spatial interactions and habitat use of rabbits on pasture and implications for the spread of rabbit haemorrhagic disease in New South Wales. Wildlife Research 30:49-58.

White, P. J., K. Ralls, and D. B. Siniff. 2000. Nocturnal encounters between kit foxes. Journal of Mammalogy 81:456-461.

\section{APPENDIX. PROCESSING PROXIMITY LOGGER DATA}

We conducted our study under simulated field conditions on live animals. Consequently, contacts might often take place near the limit of the detection distance where the signal strength was weaker and more variable. Variation from logger transmitters combined with interactions occurring near the limits of detection can cause differences in the number and duration of contacts (Prange et al. 2006). We made an ad hoc assumption that an encounter occurred if one or both loggers recorded an interaction. Proximity loggers were synchronized to a single time source.

We wrote code for R (R Development Core Team, Vienna, Austria) that would process jointly recorded encounters between pairs of deer into single encounter records. The separation time of our proximity loggers was set to $120 \mathrm{~s}$; thus, all records had $60 \mathrm{~s}$ subtracted from the start time (lower time) and added to the end time (upper time) to buffer records with 

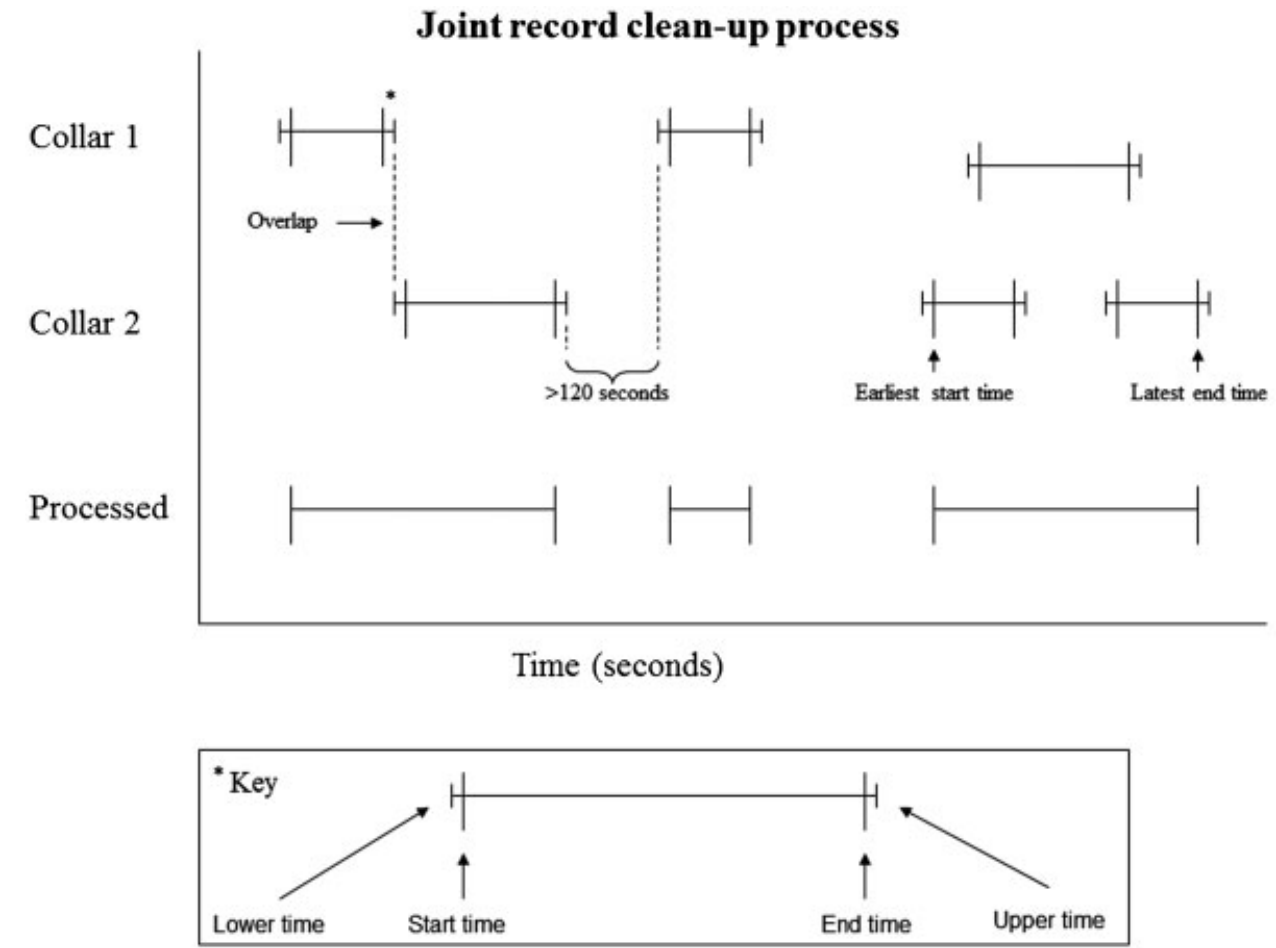

Figure A1. Procedure for processing jointly recorded encounters by proximity loggers into single encounter records. Encounters were recorded from interactions between white-tailed deer fitted with proximity loggers during a study at Sandhill Wildlife Management Area (Babcock, WI, USA) $2007-2008$.

an appropriate threshold for separation time (Fig. A1). If interactions overlapped anywhere between the lower and upper time buffers, the code would select the earliest start time and latest end time, calculate the new encounter duration and create an updated encounter record with the new start time, end time, and encounter duration (Fig. A1). The process provided us with a data set that contained full contact records for encounters between maternal pairs of deer.

Associate Editor: Rodgers. 